\title{
ALK INHIBITORS AND POSSIBLE SMALL MOLECULES OF NON-SMALL CELL LUNG CANCER: A LITERATURE REVIEW
}

\author{
Utpalendu Paul \\ School of BioScience and Technology \\ Vellore Institute of Technology (VIT), \\ Vellore, Tamil Nadu, India
}

\author{
Subhadeep Banerjee \\ School of BioScience and Technology \\ Vellore Institute of Technology (VIT), \\ Vellore, Tamil Nadu, India
}

\begin{abstract}
Lung cancer is among the deadliest cancers for humans and makes up about $13 \%$ of all diagnosed cancer. NSCLC, which accounts for $85 \%$ of all lung cancer is epithelial lung cancer and consists of four stages. Many proteins can cause NSCLC, among them is Anaplastic Lymphoma Kinase (ALK). ALK-EML-4 fusion form causes tumorigenesis. Patients with NSCLC having EML4ALK gene rearrangements are not associated with ethnicity, smoking or sex. According to this factor, many available small molecules have been developed which can inhibit ALK and therefore decreases the progression of NSCLC in patients but certain limitations of these drugs have led to the discovery of more potent small molecules. Treatment with small molecules has provided effective clinical benefit with very little toxicity. This study aims at finding potent small molecules using BindingDB according to their $\mathrm{IC}_{50}$ values to overcome the resistance of preexisting drug molecules.
\end{abstract}

Keywords-ALK, ALK Inhibitors, ALK-TKIs, BindingDB, IC50 values, NSCLC

\section{INTRODUCTION}

\section{A. Non-Small Cell Lung Cancer (NSCLC) and global statistics}

Lung cancer is among the deadliest cancers for both men and women. Over half of patients diagnosed with lung cancer have a mortality rate of $17.8 \%$. There are two main subtypes of lung cancer, Small-Cell Lung Carcinoma (SCLC) which accounts for $15 \%$ of all lung cancer and Non-Small-Cell Lung Carcinoma (NSCLC), which accounts for $85 \%$ of all lung cancer. NSCLC which is an epithelial lung cancer can be further classified into three types: Squamous-cell Carcinoma, Adenocarcinoma, and Large-cell Carcinoma. Lung cancer makes up about $13 \%$ of all diagnosed cancer [1].

Squamous-cell Carcinoma involves 25-30\% of all the lung cancer cases and arises from early versions of the squamous cells in the airway epithelial cells in the bronchial tubes in the center of the lungs. This subtype strongly corresponds to cigarette smoking. Adenocarcinoma, the most common type and comprises $40 \%$ of all lung cancer. It arises from small airway epithelial, Type II alveolar cells, which secrete mucus and other substances. It tends to grow slower and has a greater chance of being found before it has spread outside of the lungs. Large cell (undifferentiated) carcinoma accounts for $5-10 \%$ of lung cancers. This type of carcinoma shows no evidence of squamous or glandular maturation. It often begins in the central part of the lungs, near lymph nodes and into the chest wall and distant organs. Large cell carcinoma tumors are strongly associated with smoking [2] [3].

The stage of NSCLC is based on a combination of several factors size, location and metastatic property. There are five stages of NSCLC -

- $\quad$ Stage 0 - This is called in situ disease and has not grown into nearby tissues and spread outside the lung.

- $\quad$ Stage I - This stage, is a small tumor that has not spread to any lymph nodes, making it possible for a surgeon to completely remove it. It is divided into two sub-stages (IA \& IB).

- $\quad$ Stage II - It is further divided into two sub-stages IIA (tumor size $4-5 \mathrm{~cm}$ but not spread to the nearby lymph nodes) and IIB (tumor size $<5 \mathrm{~cm}$ but has spread to lymph nodes). They can be removed with surgery.

- $\quad$ Stage III - They are classified as either stage IIIA, IIIB, or IIIC. In stage IIIA and stage IIIB cancers, the tumor may be difficult or sometimes impossible, to remove with surgery. Stage IIIC cancers cannot be removed with surgery.

- $\quad$ Stage IV - It means that cancer has spread to other lung or distant parts of the body via the blood. NSCLC is more likely to spread to the brain, bones, liver, and adrenal glands. They are further classified into two sub-stages IVA (spread within the chest) and IVB (spread outside the chest).

Early-stage NSCLC has a much better diagnosis and can usually be treated radically with either surgical resection or radical radiotherapy, with favourable long-term outcomes. Along with the image-based screening method, other methods like breath-based and biofluid-based methods are currently being explored for early detection of NSCLC [4]. 
Diagnosis of lung cancer depends on the age, sex, ethnicity and geographical location of the patients. According to World Cancer Research Fund (WCRF), this disease remains the most common cancer in men worldwide (17\% of all cancers) and the third most common cancer in women ( $9 \%$ of all cancers). The highest estimates are found in Central \& Eastern Europe, Eastern Asia, Northern America and Northern Europe. The lowest incidence rates are observed in Middle \& Western Africa. The average age of diagnosis is 70. The 5-year survival rate for NSCLC is $24 \%$ which means that about $24 \%$ of the people have survived for a minimum of five years after being diagnosed with cancer. For people diagnosed with localized NSCLC (cancer has not spread outside of the lung), the overall 5 -year survival rate is $61 \%$. For regional NSCLC (cancer has spread outside of the lung to nearby areas) the 5-year survival rate is about $35 \%$. In India, lung cancer comprises of about $6.9 \%$ of all the new cancer cases and $9.3 \%$ of all cancer-related deaths in both sexes. According to the GLOBOCAN 2008 report, males predominate with a ratio (male:female) of 4.5:1 and this ratio changes with age and smoking status. The overall estimated lung cancer mortality in India in 2012 was about 63,759 thus, making it the third most common cause of cancerrelated deaths in India [5].

\section{B. Role of Anaplastic Lymphoma Kinase (ALK) in NSCLC}

The last decade has revealed the heterogeneity of molecular abnormalities present in NSCLC and thus led to the development of Tyrosine Kinase Inhibitors (TKIs), which is commonly referred to as precision oncology. Rearrangements (inversions or translocations) characterize the genomic changes in Anaplastic Lymphoma Kinase (ALK) which are observed in the NSCLC phenotype. Inversions in the short arm of Chromosome 2 that juxtapose EML4 with ALK, thus producing EML4-ALK-fusion Tyrosine Kinases. Tumors with ALK rearrangements are subjected to ALK signaling and therefore are inhibited by ALK TKIs in preclinical models [6]. Patients diagnosed with metastatic NSCLC earlier received first-line and second-line chemotherapy. Now with the discovery of Tyrosine Kinase Inhibitors (TKIs) and antibodies against specific gene mutations, the scenario of the treatment of lung cancer has changed from histologic subtype-based treatment to molecularly defined subtypes-based treatment.

Available target proteins of Non-Small Cell Lung Cancer (NSCLC) [7] are -

\section{- $\quad$ ALK (Anaplastic Lymphoma Kinase)}

- $\quad$ EGFR (Epidermal Growth Factor Receptor)

- $\operatorname{ROS1}$ (ROS proto-oncogene 1)

- HER2 (erb-b2 Receptor Tyrosine kinase 2)

- BRAF

- $\quad$ RET

- $\quad$ MET

- VEGF

\section{- $\quad$ KRAS}

- FGFR

Anaplastic Lymphoma Kinase (ALK) which is also known as ALK Tyrosine Kinase Receptor or CD246 (Cluster of Differentiation 246) is an enzyme encoded by highly conserved human alk gene. They are part of the Receptor Tyrosine Kinases (RTK) protein superfamily. ALK contains 1620 amino acid residues and has a molecular weight of $180 \mathrm{KDa}$. ALK was discovered in ALCL cells (Anaplastic Large Cell Lymphoma) in 1994. The tyrosine kinase domain shares homology with the insulin receptor [8]. ALK receptor plays a significant role in cellular communication, development and differentiation as well as in the normal function and development of the nervous system. They also regulate retinol axon targeting, synapse development at the neuromuscular junction, responses to ethanol and maintains sleep pattern.

ALKs are initially found in constitutively activated oncogenic fusion forms with EML-4 (Echinoderm Microtubule-associated Protein-like 4). Rearrangements of the ALK gene were first recognized in the year 2007 [9] and occurred in 3\% to $5 \%$ of all patients with advanced NSCLC phenotype. Whenever there is an ALK fusion, it will cause activation of the ALK protein kinase domain which plays a key role in tumorigenesis. The Cterminal domain of the fusion protein controls the protein's expression level and its activation. Therefore, these cells show uncontrolled proliferation and hence can survive, differentiate, and migrate leading to the cancer phenotype. Some of the known cancers caused by ALK fusion are - Diffuse Large Bcell Lymphomas, inflammatory myofibroblastic tumors, systemic histiocytosis, esophageal squamous cell cancers and NSCLC. Recent molecular techniques have identified ALK to be associated with neuroblastomas. Amplification of ALK locus and subsequent overexpression of ALK protein can lead to several other cancers such as - melanoma, NSCLC, glioblastoma, rhabdomyosarcoma, ovarian cancer, breast cancer, astrocytoma, Ewing's sarcoma, and retinoblastoma [10].

The constitutive expression of EML4-ALK fusion proteins and its tyrosine kinase activity can be used as a therapeutic target for most of the carcinomas, especially 5\% of all NSCLC. NSCLC caused by EML4-ALK gene rearrangements does not depend on sex, ethnicity, smoking status or various outcomes related to combination Platinum-based chemotherapy of the individual. So, targeting ALK can decrease the progression of NSCLC [11]. Crizotinib, an effective oral ALK inhibitor provides effective clinical benefit with very little toxicity in patients diagnosed with NSCLC, also the mechanism of clinical resistance to Crizotinib is identified. Other drugs such as - Ceritinib, Alectinib, Lorlatinib, etc. The use of such drugs has proved to be effective against ALK-positive non-small cell lung cancer patients. Therefore, "targeted" therapy for oncogenic proteins provides personalized medicines and evokes the mutation analysis of a wide variety of genes associated with human tumors to find possible therapeutic targets [11] [12]. 
However, these available small molecules have certain limitations and confer resistance to the available ALK inhibitors. Therefore, new strategies are in progress to overcome these limitations. Hence, this study identifies the small molecules using the BindingDB [13] dataset based on $\mathrm{IC}_{50}$ values (concentration of drug that is required for $50 \%$ inhibition) that can curb the resistance offered by already available drug molecules.

\section{PROPOSED AlgorithM}

\section{A. Literature mining of small molecules for NSCLC}

All the available small molecules for ALK-positive NSCLC have been identified through literature mining. Validated information of all the available small molecules was fetched and summarized with their year of discovery, approval status, their respective limitations and mutations along with their references.

'DrugBank' [14] (a pharmaceutical knowledge base that is enabling major advances across the data-driven medicine industry) database along with literature study was used to find the year of discovery and their approval status.

\section{B. Database search for possible small molecules for ALK- positive NSCLC}

A gold standard dataset, BindingDB [13] (a public, webaccessible database of measured binding affinities, focusing chiefly on the interactions of proteins considered to be candidate drug-targets with ligands that are small, drug-like molecules) was used to find all the possible small molecules using keywords "ALK" and "Anaplastic Lymphoma Kinase". Two separate tables were prepared accordingly.

\section{EXPERIMENT AND RESULT}

\section{A. Literature analysis of the available small molecules of ALK-positive NSCLC}

Small molecules such as Crizotinib (first-generation ALKTKIs) as well as Ceritinib, Alectinib and Brigatinib (secondgeneration ALK-TKIs) have shown remarkable clinical efficacy in ALK-rearranged NSCLC. Lorlatinib is a novel third-generation TKI which targets ALK as well as ROS1 in NSCLC diagnosed patients. However, acquired resistance to these TKIs is an inevitable and crucial limitation. Another major limitation of the conventional ALK-TKIs is that their efficiency against the Central Nervous System (CNS) metastasis is inadequate, due to the protective effect of the Blood-Brain Barrier (BBB) against toxicity. Some common side effects, toxic effects and adverse effects for all these small molecules are - Nausea, Fever, Vomiting, Diarrhea, Constipation, Fatigue, Changes in Vision, Loss of Appetite, Dizziness, problems in Heart rhythm function, Skin rashes, allergies, sensitive to the sun and swelling in lungs and other body parts. Acquired resistance to the TKIs is an inevitable and crucial limitation for all small molecules.
Crizotinib ( $\mathrm{IC}_{50}$ value of $24 \mathrm{nM}$ ) is the first-generation ATPcompetitive ligand inhibitor of the Receptor Tyrosine Kinases (RTK) C-Met, ALK and ROS1. Rearrangement of the EML4ALK gene leads to continuous activation of ALK-RTK, representing 3-5\% of NSCLC. Crizotinib is meant for this entity. It is the first drug to be approved as an ALK inhibitor on 26 August 2011. Its high efficiency in ALK rearrangement as new molecular targets underlines the significance of molecular typing in NSCLC. It has shown significant improvements in progression-free survival and overall response rate compared with standard chemotherapy and its safety profile was acceptable [15]. Some mutations confer resistance to this firstgeneration ALK-TKI. These include L1196M, C1156Y, L1152R, G1202R, S1206Y, F1174S, I1171T, G1269A, E1210K + D1203N, G1269S, L1198P, F1174L, L1152P, S1206C, I1171N, I1171S, F1174C, F1174V, V1180L and I1151Tins [16]. Limitations include [17]-

- Elevated liver aminotransferase level.

- Limited Blood-Brain Barrier penetration

- Visual Disorders \& Gastrointestinal side effects

- Relapse of Central Nervous System

Ceritinib ( $\mathrm{IC}_{50}$ value of $0.15 \mathrm{nM}$ ) is a second-generation ALK inhibitor that has shown greater antitumor potency than Crizotinib in preclinical studies. It is an oral, small-molecule, ATP-competitive, tyrosine kinase inhibitor of ALK. In various enzymatic assays, Ceritinib is found to be about 20 times as potent as Crizotinib against ALK. The therapeutic effect of Ceritinib is by inhibiting the autophosphorylation of ALK, ALK-mediated phosphorylation of the downstream signaling protein STAT3, and proliferation of ALK-dependent cancer cells. On 29 April 2014, the FDA approved Ceritinib, making a breakthrough therapy-designated drug (second-generation ALK-TKIs), for the treatment of patients with ALK-positive, metastatic NSCLC who have progressed on or are intolerant to Crizotinib [18]. Ceritinib was found to be highly active in patients with advanced, ALK-rearranged NSCLC, including those who had had disease progression during Crizotinib treatment, regardless of the presence of resistance mutations in ALK. Mutations include G1202R, F1174C, F1174V, C1156Y, L1152R, L1152P, F1174L, V1180L, I1151T, C116T, G1202del, D1203N + E1210K, F1174C + D1203N, G1123S, L1198F provides resistance to Ceritinib small molecule [19]. Limitations of Ceritinib include [20] -

- Common adverse events such as nausea, diarrhea, vomiting and fatigue along with elevated transaminases.

- Increased alanine aminotransferase

- Increased aspartate aminotransferase

- Elevated levels of lipase

Some of these toxicities were reversible after Ceritinib administration was stopped.

Brigatinib ( $\mathrm{IC}_{50}$ value of 1.5 - 12nM) second-generation ALKTKI associated with metastatic NSCLC, was approved on 28 April 2017 by FDA. It is a reversible dual inhibitor of ALK and EGFR. It shows selectivity against nine Crizotinib-resistant 
mutants of the EML4-ALK fusion gene, which is significant in the transformation of susceptible lung parenchyma. Due to the mutations in the ALK kinase domain, Crizotinib becomes resistant to the treatment. Therefore, Brigatinib is used for the treatment of patients with ALK-positive NSCLC having Crizotinib-resistance [21]. Mutations such as - E1210K, S1206C, D1203N, G1202R, F1174V, L1198F, D1203N + E1210K, F1174C + D1203N, G1202del causes resistant to Brigatinib [22]. Limitations include [23] -

- Testicular Toxicity

- Dyspnea, Hypoxia, Pneumonia

- Testicular Tubular Degeneration

- Hypertension

- Weight loss

- Hypoespermatogenesis

Alectinib ( $\mathrm{IC}_{50}$ value of $\left.1.9 \mathrm{nM}\right)$ a highly selective inhibitor of ALK, has shown systemic and central nervous system (CNS) efficacy in the treatment of ALK-positive NSCLC. It is specifically used in the treatment of non-small cell lung cancer expressing the ALK-EML4 (Echinoderm Microtubuleassociated protein-like 4) fusion protein that causes the proliferation of NSCLC cells. Its approval was granted on 11 December 2015 by the FDA for the treatment of patients with ALK-positive, NSCLC who have progressed on or are resistant to Crizotinib [24]. This small molecule is an orally biologically available TKI that inhibits ALK and RET proteins by preventing their phosphorylation. Inhibition of ALK activation prevents the downstream signaling of cell proliferation and decreases tumor survivability. Alectinib possesses five-fold more efficacy than Crizotinib in inhibiting ALK and thus helps in maintaining the activity against many secondary mutants associated with the resistance to Crizotinib. Alectinib has very good Blood-Brain Barrier penetration. Alectinib acts as an active small molecule in the majority of Crizotinib-resistant ALK-positive NSCLC patients. This small molecule was associated with lower toxicity and longer progression-free survival than Crizotinib and showed activity against CNS disease in patients with ALK-positive NSCLC. Mutations such as - V1180L, I1171T, G1202R, I1171N, I1171S, V1185L + L1196M, L1196Q, I1151S, D1203N + E1210K, F1174C + D1203N confers resistant to Alectinib [25]. Limitations of Alectinib include [20] -

- Dysgeusia,

- Increased bilirubin and creatinine levels

- Gastrointestinal symptoms.

- In the Phase II trials, the most common adverse events were constipation, fatigue, myalgia and peripheral edema.

- Aspartate aminotransferase

(AST)/alanine aminotransferase (ALT) elevation,

- Creatine phosphokinase (CPK) elevation, nausea, diarrhea, and rash.

- Reduced neutrophil count,

- Dyspnea

- Elevations in liver enzymes or CPK
Although Alectinib seems to have protective efficacy against the progression of CNS metastases, patients with CNS metastases showed CNS progression, even though treated with Alectinib. Lorlatinib ( $\mathrm{IC}_{50}$ value of $1.3 \mathrm{nM}$ ) is designed to penetrate the Blood-Brain Barrier (BBB) and therefore can overcome this limitation. Lorlatinib is more potent than Crizotinib, Alectinib or Ceritinib, having a $\mathrm{Ki}$ value of 0.11 nM. Lorlatinib was approved by FDA in November 2018 and therefore has shown significant efficiency in patients with ALK-NSCLC resistant to first and second-generation ALKTKIs also possesses antitumor property against CNS metastasis attributable to high $\mathrm{BBB}$ permeability. Interestingly, DNA sequential analysis after resistance to Lorlatinib revealed the double mutation ALK C1156Y/L1198F, which might represent the cause of Lorlatinib resistance and could be successfully targeted by Crizotinib. Lorlatinib resistance is mediated by multiple different compound ALK mutations, known as double ALK mutations. The ALK G1202R/L1196M mutant is resistant to all ALK-TKIs, while ALK C1156Y/L1198F is resistant to next-generation inhibitors including Lorlatinib, but paradoxically is sensitive to Crizotinib [26]. Other mutations include L1198F, L1256F, L1198H, L1204V, G1269A, F1174V, F1174C, F1174I, F1174L, D1203N, I1171T, I1171N, I1171S, I1179V, E1210K, S1256F, G1202R + L1196M [27]. Limitations include [28] -

\section{- Hypercholesterolemia}

- Peripheral neuropathy, Edema, Increased Weight

- Hypertriglyceridemia, Fatigue

- Gastro-Intestinal side effects

- Increased lipase

- Embryo-fetal harm when administered to a pregnant woman

- Ineffectiveness of hormonal contraceptives

- Atrophy

- Epididymal inflammation

Entrectinib ( $\mathrm{IC}_{50}$ value of $12 \mathrm{nM}$ ) is a small-molecule TKI that targets neurotrophic tyrosine kinase receptor ALK. Several clinical trials of Entrectinib in advanced or metastatic cancer are being conducted. It was approved by the FDA in August 2019 for its use in the treatment of ROS1-positive metastatic NSCLC patients. However, the efficacy of Entrectinib (in Crizotinib-resistant) is questionable because in preclinical studies' results it did not show much activity against the ALK G1202R mutation, which is a common Crizotinib-resistant mutation [29]. Mutations such as R1275Q, F1174L, F1245C causes resistance to the small molecule [30]. Limitations include [31] -

- Myalgia, arthralgia, QT prolongation

- Increased secondary infections

- Paresthesia

- Dysgeusia, weight gain

Ensartinib ( $\mathrm{IC}_{50}$ value of $<4 \mathrm{nM}$ ) is a novel potent ALK-TKI engineered to inhibit two ALK point mutations (L1996Mand C1556Y) known to be Crizotinib-resistant mutations. This drug is in the investigational stage and has not been approved yet by 
the FDA. Phase I/II trial [NCT01625234] of Ensartinib demonstrated good clinical activity and tolerability in patients with NSCLC having ALK mutation. A randomized phase III trial is ongoing comparing the efficacy of Ensartinib and Crizotinib in treatment-naive ALK-positive patients [26]. The Phase III Trial data comparing Ensartinib with Crizotinib (enrollment was closed in 2018) are being awaited. Beyond the clinical results, a pre-clinical activity of this small molecule is reported against some ALK mutations such as - $\mathrm{C} 1156 \mathrm{Y}$, L1196M, S1206R, F1174 and T1151 mutants while it does not seem to be active against ALK G1202R mutation. Limitations include [32] -

- $\quad$ Fatigue, nausea, vomiting

- Edema, Pruritus, Decreased appetite, urinary tract infection

- Diarrhea, constipation, Anemia, hypertension

Repotrectinib is a next-generation ALK inhibitor that was designed to overcome the acquired resistance because of the mutations - ALK G1202R. This small molecule basically targets ROS1-TKI with improved potency, but its action as an ALK inhibitor is also being studied and therefore is in the experimental stage and has not yet been approved by the FDA. According to the study, Repotrectinib shows a durable and ongoing response in Crizotinib-refractory patients harboring the ALK G1202R mutation. The result of a Phase I trial [NCT03093116] showed that this drug is well tolerated in patients. Clinical activity data is warranted in the future [33].

Small molecules, CEP28122 is in pre-clinical studies and CEP37440 is in Phase I clinical development.

CEP-28122 ( $\mathrm{IC}_{50}$ value of $\left.1.9 \mathrm{nM}\right)$ is a potent and selective ALK inhibitor. In the preclinical studies, this small molecule showed high selectivity against ALK among other tyrosine kinases, including InsR, IGF-R1 and cMET [34].

CEP37440 is an inhibitor of ALK and Focal Adhesion Kinase (FAK). CEP37440 is undergoing clinical development in Phase I [NCT01922752] in patients with advanced or metastatic solid tumors but no preliminary data are available [34].

The other two small molecules are known to act as inhibitors of ALK. They are - ASP3026 and TSR-011.

ASP3026, second-generation ALK-TKI, is a significant antitumor activity against wild-type ALK and EML4-ALK (L1196M) xenograft tumors compared with Crizotinib. Preclinical data showed that ASP3026 may have potential therapeutic effects in Crizotinib-resistant ALK-positive NSCLC patients. Another ALK mutation I1171T causes resistance to ASP3026 [25]. Adverse events such as Neutropenia (Febrile), thrombocytopenia, non-hematologic toxicity, Keratoacanthoma, increased aspartate aminotransferase, drug eruption, etc. along with one death of ASP3026-administered patient and other strategic reasons led to the withdrawal of the drug [35].

TSR-011 is significant for dose-dependent concentrations (nM) against wild-type ALK in vitro, ALK-dependent cellular models and in ALK-dependent tumor growth inhibitory activity in mouse models. Besides, TSR-011 is around 200-fold more efficient than Crizotinib against the L1196M ALK mutant form. Adverse events like Anaemia, Asthenia, ECG QTc prolongation, pneumonitis, Dyspnoea, Ascites, fatigue, CNS metastases, etc. led to the discontinuation of drug administration [36].

\section{A. Selection of the possible small molecules for ALK- positive NSCLC}

In BindingDB, the searched keyword "ALK" gave about 5338 small molecules and the keyword "Anaplastic Lymphoma Kinase" gave about 2829 small molecules. After removing redundancy, a final spreadsheet was prepared where 522 small molecules have been identified based on their $\mathrm{IC}_{50}$ values $<5.0$ which can act as possible ALK Inhibitors. The searched keywords are listed in the table below.

Table - 1 Search result of ALK from BindingDB

\begin{tabular}{|c|c|c|c|}
\hline $\begin{array}{l}\text { Sr. } \\
\text { No. }\end{array}$ & Keyword & Title & Compounds \\
\hline \multirow{7}{*}{1.} & \multirow{7}{*}{ ALK } & $\begin{array}{l}\text { ALK Tyrosine } \quad \text { Kinase } \\
\text { Receptor }\end{array}$ & 2462 \\
\hline & & $\begin{array}{lll}\text { ALK } & \text { Tyrosine } & \text { Kinase } \\
\text { Receptor } & \text { (ALK) } & \end{array}$ & 2456 \\
\hline & & $\begin{array}{lcc}\text { ALK } & \text { Tyrosine } & \text { Kinase } \\
\text { Receptor } & (\text { F1174L) } & \end{array}$ & 2 \\
\hline & & $\begin{array}{lll}\text { ALK } & \text { Tyrosine } & \text { Kinase } \\
\text { Receptor }(\text { L1196M) } & \end{array}$ & 359 \\
\hline & & $\begin{array}{lcl}\text { ALK } & \text { Tyrosine } & \text { Kinase } \\
\text { Receptor } & (\text { R1275Q) } & \end{array}$ & 2 \\
\hline & & $\begin{array}{lll}\text { ALK } & \text { Tyrosine } & \text { Kinase } \\
\text { Receptor } & (\mathbf{C} 1156 Y) & \end{array}$ & 2 \\
\hline & & $\begin{array}{l}\text { ALK } \\
\text { Receptor/Nucleophosmin }\end{array}$ & 55 \\
\hline \multirow{3}{*}{2.} & \multirow{3}{*}{$\begin{array}{l}\text { Anaplastic } \\
\text { Lymphoma } \\
\text { Kinase }\end{array}$} & $\begin{array}{ll}\text { Anaplastic } & \text { Lymphoma } \\
\text { Kinase } & \end{array}$ & 2462 \\
\hline & & $\begin{array}{lr}\text { Anaplastic } & \text { Lymphoma } \\
\text { Kinase } & \text { C1156Y } \\
\text { C1156Y) } & \end{array}$ & 8 \\
\hline & & $\begin{array}{l}\text { Anaplastic } \begin{array}{r}\text { Lymphoma } \\
\text { Kinase } \\
\text { L1196M) }\end{array} \\
\text { L1196M }\end{array}$ & 359 \\
\hline
\end{tabular}

\section{CONCLUSION}

The lethality and universality of NSCLC highlight the importance of identifying ALK inhibitors for the treatment of ALK-positive NSCLC. From the recent success of Crizotinib and Ceritinib with very little toxicity and effective clinical benefit of NSCLC patients, it is predicted that targeted therapy can give possible outcomes. Although Crizotinib being identified as a potent ALK inhibitor, most of the patients treated with the same had acquired resistance. To overtake these acquired resistances to Crizotinib, prolong the control of 


\section{International Journal of Engineering Applied Sciences and Technology, 2020 \\ Vol. 5, Issue 8, ISSN No. 2455-2143, Pages 158-164 \\ Published Online December 2020 in IJEAST (http://www.ijeast.com)}

the disease several 2nd and 3rd generation TKIs - Alectinib, Brigatinib, Lorlatinib, etc. have been developed.

However, these available ALK inhibitors have limitations and certain mutations (i.e. L1196M, G1269A, F1174L, C1156Y, etc.) in ALK are responsible for the acquired resistance against these available ALK inhibitors [37]. Further studies can be performed to determine more mutations in ALK so that more potent inhibitors can be developed.

The use of BindingDB for ALK inhibitors can give several small molecules other than the available ones which can be grouped according to their IC50 values (concentration of drug that is required for $50 \%$ inhibition). Further Docking and Modelling studies can be performed to develop these small molecules into potent drug molecules that can overcome the acquired resistance so that they can inhibit ALK and hence attenuate NSCLC along with the possibility of a cure.

\section{REFERENCE}

[1] F. R. Hirsch et al., "Lung cancer: current therapies and new targeted treatments," Lancet, vol. 389, no. 10066, pp. 299-311, 2017, doi: 10.1016/S01406736(16)30958-8.

[2] C. Zappa and S. A. Mousa, "Non-small cell lung cancer: Current treatment and future advances," Transl. Lung Cancer Res., vol. 5, no. 3, pp. 288-300, 2016, doi: 10.21037/tlcr.2016.06.07.

[3] L. A. Pikor, V. R. Ramnarine, S. Lam, and W. L. Lam, "Genetic alterations defining NSCLC subtypes and their therapeutic implications," Lung Cancer, vol. 82, no. 2, pp. 179-189, 2013, doi: 10.1016/j.lungcan.2013.07.025.

[4] H. Balata et al., "Prevention and Early Detection for NSCLC: Advances in Thoracic Oncology 2018," $J$. Thorac. Oncol., vol. 14, no. 9, pp. 1513-1527, 2019, doi: 10.1016/j.jtho.2019.06.011.

[5] P. Parikh et al., "Lung cancer in India: Current status and promising strategies," South Asian J. Cancer, vol. 5, no. 3, p. 93, 2016, doi: 10.4103/2278-330x.187563.

[6] D. B. Costa et al., "Clinical experience with crizotinib in patients with advanced ALK-rearranged non-smallcell lung cancer and brain metastases," J. Clin. Oncol., vol. 33, no. 17, pp. 1881-1888, 2015, doi: 10.1200/JCO.2014.59.0539.

[7] Q. G. Zhu, S. M. Zhang, X. X. Ding, B. He, and H. Q. Zhang, "Driver genes in non-small cell lung cancer: Characteristics, detection methods, and targeted therapies," Oncotarget, vol. 8, no. 34, pp. 5768057692, 2017, doi: 10.18632/oncotarget.17016.

[8] K. Kinoshita, N. Oikawa, and T. Tsukuda, Anaplastic Lymphoma Kinase Inhibitors for the Treatment of ALK-Positive Cancers, 1st ed., vol. 47. Elsevier Inc., 2012.

[9] T. Li, W. Ma, and E. C. Tian, "Ensartinib (X-396): What does it add for patients with ALK-rearranged
NSCLC," Chinese Clin. Oncol., vol. 8, pp. 8-12, 2019, doi: 10.21037/cco.2018.10.12.

[10] T. R. Webb et al., "Anaplastic lymphoma kinase: Role in cancer pathogenesis and small-molecule inhibitor development for therapy," Expert Rev. Anticancer Ther., vol. 9, no. 3, pp. 331-356, 2009, doi: 10.1586/14737140.9.3.331.

[11] A. Manuscript, "to Therapy in Record Time," vol. 18, no. 6, pp. 548-551, 2011, doi: 10.1016/j.ccr.2010.11.033.ALK.

[12] P. M. Forde and D. S. Ettinger, "Targeted therapy for non-small-cell lung cancer: Past, present and future," Expert Rev. Anticancer Ther., vol. 13, no. 6, pp. 745758, 2013, doi: 10.1586/era.13.47.

[13] T. Liu, Y. Lin, X. Wen, R. N. Jorissen, and M. K. Gilson, "BindingDB: A web-accessible database of experimentally determined protein-ligand binding affinities," Nucleic Acids Res., vol. 35, no. SUPPL. 1, pp. 198-201, 2007, doi: 10.1093/nar/gk1999.

[14] D. S. Wishart et al., "DrugBank 5.0: A major update to the DrugBank database for 2018," Nucleic Acids Res., vol. 46, no. D1, pp. D1074-D1082, 2018, doi: 10.1093/nar/gkx1037.

[15] D. F. Heigener and M. Reck, "Crizotinib," pp. 57-65, doi: 10.1007/978-3-319-91442-8.

[16] J. M. Heuckmann et al., "ALK mutations conferring differential resistance to structurally diverse ALK inhibitors," Clin. Cancer Res., vol. 17, no. 23, pp. 7394-7401, 2011, doi: 10.1158/1078-0432.CCR-111648.

[17] R. Pirker and M. Filipits, "From crizotinib to lorlatinib: Continuous improvement in precision treatment of ALK-positive non-small cell lung cancer," ESMO Open, vol. 4, no. 5, pp. 1-4, 2019, doi: 10.1136/esmoopen-2019-000548.

[18] S. Khozin et al., "FDA approval: Ceritinib for the treatment of Metastatic Anaplastic lymphoma Kinasepositive non-small cell lung cancer," Clin. Cancer Res., vol. 21, no. 11, pp. 2436-2439, 2015, doi: 10.1158/1078-0432.CCR-14-3157.

[19] L. Friboulet et al., "The ALK inhibitor ceritinib overcomes crizotinib resistance in non-small cell lung cancer," Cancer Discov., vol. 4, no. 6, pp. 662-673, 2014, doi: 10.1158/2159-8290.CD-13-0846.

[20] M. M. Awad and A. T. Shaw, "ALK inhibitors in nonsmall cell lung cancer: Crizotinib and beyond," Clin. Adv. Hematol. Oncol., vol. 12, no. 7, pp. 429-439, 2014.

[21] S. Rashdan and D. E. Gerber, "A crowded, but still varied, space: Brigatinib in anaplastic lymphoma kinase-rearranged non-small cell lung cancer," Transl. Cancer Res., vol. 6, no. 10, pp. S78-S82, 2017, doi: 10.21037/tcr.2017.02.12.

[22] J. Li, Y. Huang, M. Wu, C. Wu, X. Li, and J. Bao, "Structure and energy based quantitative missense 


\section{International Journal of Engineering Applied Sciences and Technology, 2020 \\ Vol. 5, Issue 8, ISSN No. 2455-2143, Pages 158-164 \\ Published Online December 2020 in IJEAST (http://www.ijeast.com)}

variant effect analysis provides insights into drug resistance mechanisms of anaplastic lymphoma kinase mutations," Sci. Rep., vol. 8, no. 1, pp. 1-15, 2018, doi: 10.1038/s41598-018-28752-9.

[23] T. Beardslee and J. Lawson, "Alectinib and Brigatinib: New Second-Generation ALK Inhibitors for the Treatment of Non-Small Cell Lung Cancer.," J. Adv. Pract. Oncol., vol. 9, no. 1, pp. 94-101, 2018.

[24] E. Larkins et al., "FDA approval: Alectinib for the treatment of metastatic, ALK-positive non-small cell lung cancer following crizotinib," Clin. Cancer Res., vol. 22, no. 21, pp. 5171-5176, 2016, doi: 10.1158/1078-0432.CCR-16-1293.

[25] R. Katayama et al., "Two novel ALK mutations mediate acquired resistance to the next-generation ALK inhibitor alectinib," Clin. Cancer Res., vol. 20, no. 22, pp. 5686-5696, 2014, doi: 10.1158/10780432.CCR-14-1511.

[26] T. Akamine et al., "Lorlatinib for the treatment of patients with non-small cell lung cancer," Drugs of Today, vol. 55, no. 2, pp. 107-116, 2019, doi: 10.1358/dot.2019.55.2.2927983.

[27] S. Yoda et al., "HHS Public Access," vol. 8, no. 6, pp. 714-729, 2019, doi: 10.1158/2159-8290.CD-171256.Sequential.

[28] A. T. Shaw et al., "Lorlatinib in non-small-cell lung cancer with ALK or ROS1 rearrangement: an international, multicentre, open-label, single-arm firstin-man phase 1 trial," Lancet Oncol., vol. 18, no. 12, pp. 1590-1599, 2017, doi: 10.1016/S14702045(17)30680-0.

[29] Z. T. Al-Salama and S. J. Keam, "Entrectinib: First Global Approval," Drugs, vol. 79, no. 13, pp. 14771483, 2019, doi: 10.1007/s40265-019-01177-y.

[30] H. L. Pacenta and M. E. Macy, "Entrectinib and other ALK/TRK inhibitors for the treatment of neuroblastoma," Drug Des. Devel. Ther., vol. 12, pp. 3549-3561, 2018, doi: 10.2147/DDDT.S147384.

[31] D. Liu, M. Offin, S. Harnicar, B. T. Li, and A. Drilon, "Entrectinib: An orally available, selective tyrosine kinase inhibitor for the treatment of NTRK, ROS1, and ALK fusion-positive solid tumors," Ther. Clin. Risk Manag., vol. 14, pp. 1247-1252, 2018, doi: 10.2147/TCRM.S147381.

[32] L. Horn et al., "Ensartinib (X-396) in ALK-positive non-small cell lung cancer: Results from a first-inhuman phase I/II, multicenter study," Clin. Cancer Res., vol. 24, no. 12, pp. 2771-2779, 2018, doi: 10.1158/1078-0432.CCR-17-2398.

[33] M. R. Yun et al., "Repotrectinib Exhibits Potent Antitumor Activity in Treatment-Naïve and SolventFront-Mutant ROS1-Rearranged Non-Small Cell Lung Cancer," Clin. Cancer Res., vol. 26, no. 13, pp. 32873295, 2020, doi: 10.1158/1078-0432.CCR-19-2777.

[34] I. Sullivan and D. Planchard, "ALK inhibitors in non- small cell lung cancer: The latest evidence and developments," Ther. Adv. Med. Oncol., vol. 8, no. 1, pp. 32-47, 2016, doi: 10.1177/1758834015617355.

[35] T. Li et al., "First-in-human, open-label doseescalation and dose-expansion study of the safety, pharmacokinetics, and antitumor effects of an oral ALK inhibitor ASP3026 in patients with advanced solid tumors," J. Hematol. Oncol., vol. 9, no. 1, pp. 19, 2016, doi: 10.1186/s13045-016-0254-5.

[36] C. C. Lin et al., "A phase 1, open-label, doseescalation trial of oral TSR-011 in patients with advanced solid tumours and lymphomas," Br. J. Cancer, vol. 121, no. 2, pp. 131-138, 2019, doi: 10.1038/s41416-019-0503-9.

[37] A. Kumar, V. Shanthi, and K. Ramanathan, "Discovery of potential ALK inhibitors by virtual screening approach," 3 Biotech, vol. 6, no. 1, pp. 112, 2016, doi: 10.1007/s13205-015-0336-z. 